\title{
Enhancement Mode GaN-FETs in Extreme Temperature Conditions, Part II: Dynamic Parasitic Parameters
}

Duraij, Martijn S.; Xiao, Yudi; Zsurzsan, Gabriel; Zang, Zhe

Published in:

Additional Conferences (device Packaging, Hitec, Hiten, and Cicmt)

Link to article, DOI:

10.4071/2380-4491.2021.HiTEC.000053

Publication date:

2021

Document Version

Peer reviewed version

Link back to DTU Orbit

Citation (APA):

Duraij, M. S., Xiao, Y., Zsurzsan, G., \& Zang, Z. (2021). Enhancement Mode GaN-FETs in Extreme

Temperature Conditions, Part II: Dynamic Parasitic Parameters. Additional Conferences (device Packaging,

Hitec, Hiten, and Cicmt), 2021(HiTEC), 000053-000057. https://doi.org/10.4071/2380-4491.2021.HiTEC.000053

\section{General rights}

Copyright and moral rights for the publications made accessible in the public portal are retained by the authors and/or other copyright owners and it is a condition of accessing publications that users recognise and abide by the legal requirements associated with these rights.

- Users may download and print one copy of any publication from the public portal for the purpose of private study or research.

- You may not further distribute the material or use it for any profit-making activity or commercial gain

- You may freely distribute the URL identifying the publication in the public portal 


\title{
Enhancement Mode GaN-FETs in Extreme Temperature Conditions, Part II: Dynamic Parasitic Parameters
}

\author{
Martijn S. Duraij, Yudi Xiao, Gabriel Zsurzsan, and Zhe Zang \\ Department of Electrical Engineering \\ Technical University of Denmark \\ Kgs. Lyngby, Denmark \\ msdu@elektro.dtu.dk
}

\begin{abstract}
Parasitic components in eGaN-FETs impact the dynamic performance of switching stages. The capacitances seen, primarily on the output characteristics, of these devices are a main contributor towards switching losses and therefor converter efficiency. Additionally, the threshold voltage of the device has an impact towards the switching speed and therefore the efficiency of a power stage. This study shows the impact of extreme temperatures towards the parasitics that impact the switching behaviour of a power stage. A literature research is conducted exploring the various mechanisms and temperature dependancies, which are then related towards transient operations of eGaN-FETs. A device was chosen to perform measurements on output-, reverse transfer capacitance and threshold voltage while increasing temperature from $100^{\circ} \mathrm{C}$ op to $225^{\circ} \mathrm{C}$. The presented results show a large impact of temperature in these parasisic elements that show that high temperature switch-mode power converters need additional design work to ensure switching performance and lifetime.
\end{abstract}

Key words

Device characterization; eGaN-FET; High temperature; Parasitic components; Switching losses

\section{Introduction}

High temperature applications of power converters gain more and more attention. With goals to create smaller power converters to supply higher output powers the power per volume figure of merit (FOM) is extended. With such challenges the operating limits of all components are explored. Particulary in the industries working with elevated ambient temperatures like geothermal, oil and gas, aeronautical and military applications these topics see a trend towards higher operating temperatures. For oil and gas applications these ambient temperatures can reach up to $200^{\circ} \mathrm{C}$ [1].

Increased temperature in electronics has an impact on the full circuit containing resistors, capacitors, inductors, active devices, etc. The importance of studying the used components in the operation stage of the envisioned life cycle of the product is paramount to estimate the lifetime $[2,3]$. Temperature impacts the expected lifetime of electronics, often the expected lifetime is highly degraded when operating temperatures are increased. For active devices, such as transistors and diodes, normally operate in alternate modes of conduction/blocking currents. Hence the dynamic aspects of these components form a critial topic to understand.

For any switch-mode power converter it is important to evaluate the losses that are generated in the circuit. Lower losses lead to higher efficiencies and may lead tighter packed converters $[4,5]$. For switch-mode power converters these losses are primarily catogorized into two topics: switching losses and conduction losses. The conduction losses in devices are generated when a switching device is in a steady state, i.e. fully on or off. Parasitic components as residual on-state resistance in the channel $\left(R_{D S o n}\right)$ or leakage currents in the gate or drain $\left(I_{G S S}\right.$ and $\left.I_{D S S}\right)$ contribute to these losses. The effect of temperature on these parasitic components is discussed in [6].

This paper concentrates on the effect of temperature on parasitic components in enhancement mode Gallium-Nitride Field Effect Transistors (eGaN-FETs) that could impact switching performance. The parasitic components in these wide bandgap devices are categorized as a dynamic type, since they operate in the transient periods present in a switching stage. This means that primarily the output characteristics of the eGaN-FET will be addressed. For example: the capacitance seen in the eGaN-FET has an impact on the voltage response in the switch-node and the current in the devices. Large $d v / d t$ and $d i / d t$ transients may cause large voltage and current spikes. These spikes can damage devices or may lead to unwanted behavior in the switching stage.

Temperature has a large influence on the performance of switching devices which find their source in parasitic components. Two well nnown sources are the drift in $R_{D S o n}$ and the 
threshold voltage $V_{G S(T H)}$ over temperature, lesser is known about the influence of temperature towards capacitance in the device. The output capacitance $C_{O S S}$ and reverse transfer capacitance $C_{R R}$ are of high importance to the design of an efficient converter. The effect of temperature on the behaviour of the 2DEG in an eGaN-FET leads to an increase of this capacitance when the device heats up. The threshold voltage of the eGaNFET is also expected to be impacteded by extreme temperature conditions, as it is known to decrease with increased temperatures [7]. This will have an impact towards layout of the gate driver loop as well as the switching performance of the device.

The interest in GaN devices has been increased over the past decade and various manufactures have made their GaN-FETs available at popular vendors. This paper therefore tries to investigate performance of eGaN-FETs that are available on the market for temperatures that are exceeding the recommended operating temperatures. The selected eGaN-FETs are bare die components which allow for better GaN material performance measurments, as impact/effect from packaging is heavily reduced. It must be noted that this work does not investigate any reliability parameters of this device/these devices based on prolonged high temperature or switch-mode operation.

The first section describes the outline of this paper with background information and literature research. After that the capacitance behavior and background of eGaN-FETs is described. The effects towards switching stage performance, together with threshold voltage is discussed in the following section. This is followed by the presentation of measurement methods where after the measurement results are presented. The conclusion of this paper will be presented in the last section.

\section{Capacative behavior in high temperature}

The output capacitance $C_{O S S}$ of an eGaN-FET is the sum of the drain source capacitance $\left(C_{D S}\right)$ and the gate drain capacitance $\left(C_{G D}\right)$, the latter is also known as the reverse transfer capacitance $(C R R)$ or miller capacitance. A capacitance between the drain and the substrate is not shown because for this study the substrate is connected to the source terminal, hence it appears in parrallel with $C_{D S}$. These capacitive elements form the main contributors to the dynamic behavior of the eGaN-FETs. The output capacitance of a FET is known to be non-linear to the applied drain-source voltage across the device.

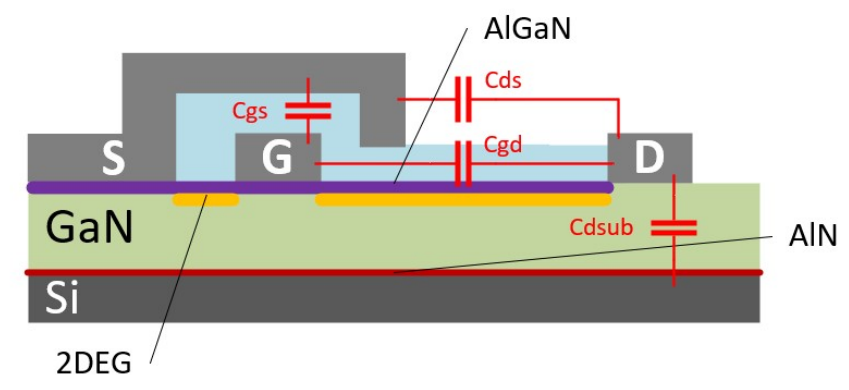

Figure 1: Structure of enhancement mode GaN-FET with placement of parasitic capacitances.

Temperature effects on these devices have been estimated and measured up to temperatures of $150{ }^{\circ} \mathrm{C}$ by [8-10]. These measurements show that an increase of capacitance is expected. However, the capacitance will still show a declining trend when a voltage is applied from drain to source. The goal of this study is to investigate if this drop-point (or knee-point) will be moved towards higher voltages. For reference in this paper this drop-off point will be defined where capacitance is decreased by $20 \%$ of its value measured at $0 \mathrm{~V}$.

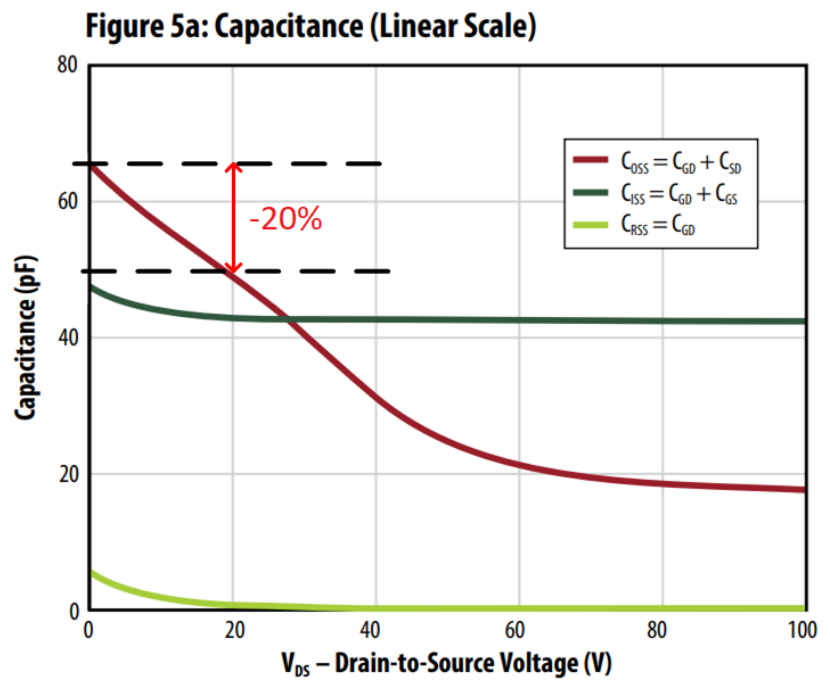

Figure 2: Example of eGaN-FET output capacitance from [7] with emphasized drop-off point.

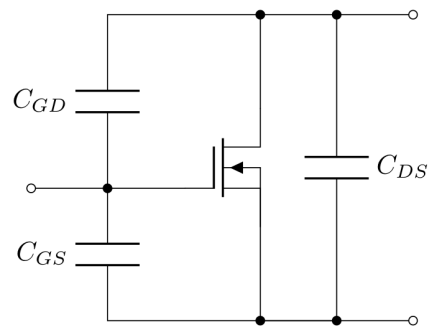

Figure 3: Parasitic capacitance in an eGaN-FET

\section{Impact of parasitics on switching behavior}

Increasing capacitance in the device structure when temperature is increased leads to a larger storage or energy between the terminals. When switching from the OFF to the ON state, this capacitance is emptied through the switch channel, in other words: shorted. This is a common behaviour known as hard switching. In high temperature circuits the available headroom in tolerated device dissipation is far lower than for regular room temperature applications. At high temperatures a designer might want 
to achieve Zero Voltage Switching (ZVS). In this practice the output capacitance of a switching element is fully discharged by ripple currents at the moment of switching and therefore reducing switching losses to zero.

An eGaN-FET is driven by applying a gate-source voltage $V_{G S}$ from the gate to the source terminal of the device. When this voltage rises above the threshold of the device $\left(V_{G S t h}\right)$ the device will start conducting current in the $2 \mathrm{DEG}$ channel. The point at which this happens is expected to be impacted by temperature. A higher temperature in the device can lead to a higher threshold voltage as is shown by [11]. The measurement shown temperatures up to $120^{\circ} \mathrm{C}$ but it is unclear if this trend will proceed with temperatures up to $225^{\circ} \mathrm{C}$.

When the device is turned ON, the left over charge in the output capacitance $C_{O S S}$ is shorted and will result in a current spike leaving the device through the source terminal. An added source impedance can impact this OFF state and may lead towards an accidental turn-ON event. For the selected device the effect towards this accidental turn-off is largely minimized in the absence of packaging of the device. Designing for a very small driving loop lowers the inductance in the gate-source path and therefore the induced voltage errors during hard switching applications.

\section{Measurement methods}

Measurements of capacitance are performed using the B1505A equipped with a Multi Frequency Capacitance Measuement Unit (MFCMU). This unit can inject a high frequency signal of $1 \mathrm{MHz}$ into the measurement node connected to the DUT using a N1260A High Voltage Bias Tee. This module allows the connection of an High Voltage Source Monitor Unit (HVSMU) to inject a DC voltage at the same time. The output capacitance and reverse transfer capacitance can then be measured versus the DC injected operating point of the drain-source voltage. For calibration a null measurement is performed to compensate for the measurement harness into the temperature chamber.

Measurement of the threshold voltage is made by shorting the gate and the drain together. A test setup is made where the gate-source voltage is gradually increased while the drain current is measured. The point at which the current starts to flow through the device is the moment that the 2DEG in the eGaN FET starts conducting and the device is starting to turn on. Normally this trigger point is set at $I_{D S}=0.25 \mathrm{~mA}$.

To create a stable high temperature environment an oven was built using aerated contrete contructions blocks and heating element from a domestic tabletop oven. Using an Arduino Uno module to set up a PWM control scheme in combination with a Solid State Relay (SSR) result in a highly stable temperature of the device to perform measurements on. The same oven is used for static parasitic component measuements in [6].

\section{Results}

The main parameter of interest in this paper is the output capacitance, where the measurement results are plotted in Fig. 4. One can observe that the output capacitance follows the expected nonlinearity when plotted versus the drain to source DC operating voltage. For low $V_{D S}$ the capacitance is high, where after this drops towards higher $V_{D S}$. For $100{ }^{\circ} \mathrm{C}$ the measured capacitance is $42 \mathrm{pF}$ at $V_{D S}=50 \mathrm{~V}$. The datasheet [7] notes a specified value of maximum $36 \mathrm{pF}$ at $100{ }^{\circ} \mathrm{C}$ which results in a difference of $16 \%$. When temperature is further increased, the capacitance also becomes larger to $47 \mathrm{pF}$ at $50 \mathrm{~V}$ in $225^{\circ} \mathrm{C}$. This results to a $30 \%$ increase compared to the datasheet values.

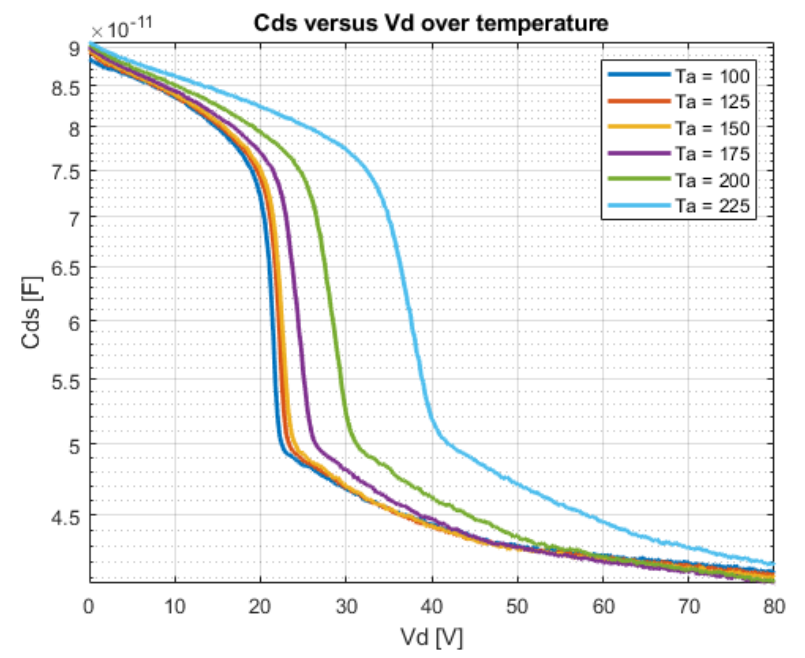

Figure 4: Measured output capacitance in selected eGaN-FET for various temperatures

More interestingly is the behaviour when temperature of the device is increased. Up to around $150{ }^{\circ} \mathrm{C}$ the curve does not change much for the measured device. Above this level, however, the capacitance sees a significant increase. When comparing results between the dark blue curve at $100{ }^{\circ} \mathrm{C}$ with the dark red curve at $225^{\circ} \mathrm{C}$, an increase of the drop-off point can be observed. Respecively the drop-off point is moving from $20.24 \mathrm{~V}$ in $100^{\circ} \mathrm{C}$, to $22.75 \mathrm{~V}$ in $175^{\circ} \mathrm{C}$ up to $34.50 \mathrm{~V}$ in $225^{\circ} \mathrm{C}$.

Another way to estimate the total capacitance increment is to integrate the plotted curve and find the average over a voltage window of $50 \mathrm{~V}$ as described in Eq. 1. When comparing the measurements, one can find a mean capacitance of $62.4 \mathrm{pF}$ in $100{ }^{\circ} \mathrm{C}$ compared to $74.1 \mathrm{pF}$ in $100{ }^{\circ} \mathrm{C}$. That is a $18.7 \%$ increase.

$$
\overline{C_{o s s}}=\frac{1}{V_{D S}} \cdot \int_{0}^{V_{D S}} C_{O S S}\left(V_{D S}\right) d V
$$

For a device that is rated at a $V_{D S}=100 \mathrm{~V}$, where the likely operation conditions can be expected right in this voltage range, the output capacitance sees a significant increase in high temperatures. This may cause trouble due to switching losses when a power stage is not designed with the right timing conditions. Excessive losses may occur and therefore highly impact the oper- 
ating performance and lifetime if this eGaN-FET was employed at these harsh conditions.

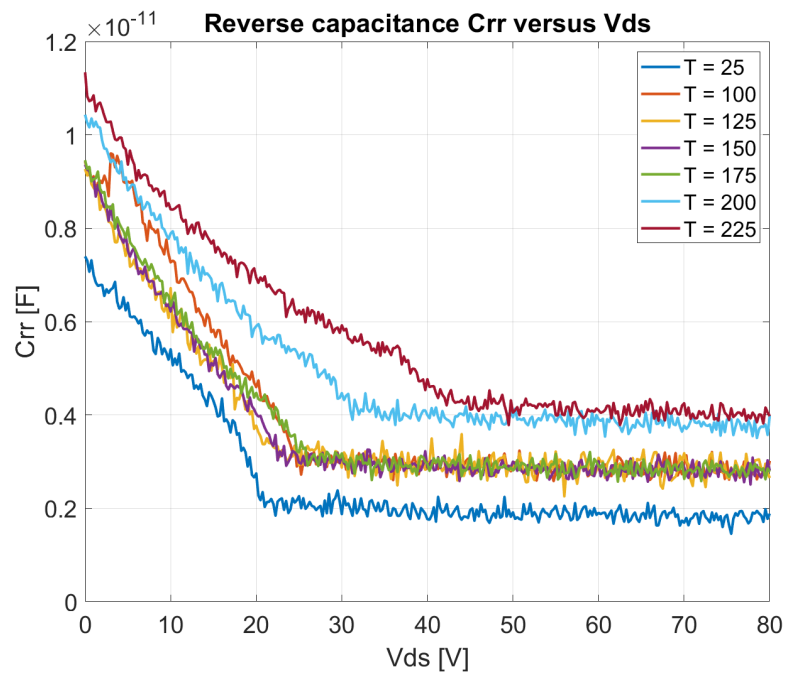

Figure 5: Measured reverse transfer capacitance in the selected eGaN-FET for various temperatures

A similar effect can be observed in Fig. 5. Temperature has a significant effect on the measured reverse transfer capacitance as well which become more of interest in the case of a turn-OFF event. The measurement error in this particular case is much larger, where the datasheet notes a maximum of $0.5 \mathrm{pF}$, measurements show $2 \mathrm{pF}$ at the lowest at $25^{\circ} \mathrm{C}$. As mentioned before capacitances are very small where measurement errors will have a large impact.

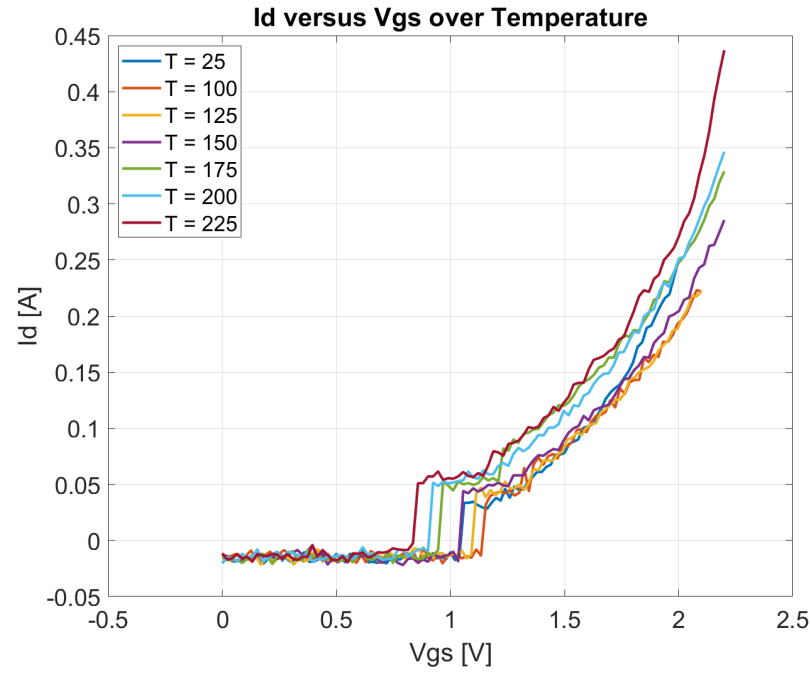

Figure 6: Measured drain current $\left(I_{D}\right)$ versus gate source voltage $\left(V_{G S}\right)$ in the selected eGaN-FET for various temperatures

The threshold voltage $V_{G S T H}$ also shows drift when exposed to various temperature conditions. The $V_{G S T H}$ can be observed when looking at the step present in Fig. 6. For each temperature, this step is displaced with $V_{G S}$, therefore emphasizing a drift in the threshold voltage. In Fig. 7 the effect over temperature is made more visible. In correlation with the works on [11] for eGaN-FETs, it is found that the threshold voltage is increasing towards higher temperatures. However, this is only valid up to $100^{\circ} \mathrm{C}$. Previous work showed a steady increase towards $120^{\circ} \mathrm{C}$ where this particular device is showing a decreasing $V_{G S T H}$ for temperatures larger than $100^{\circ} \mathrm{C}$. The reason for this drop is yet unclear, this may also be highly dependent on the chosen devices.

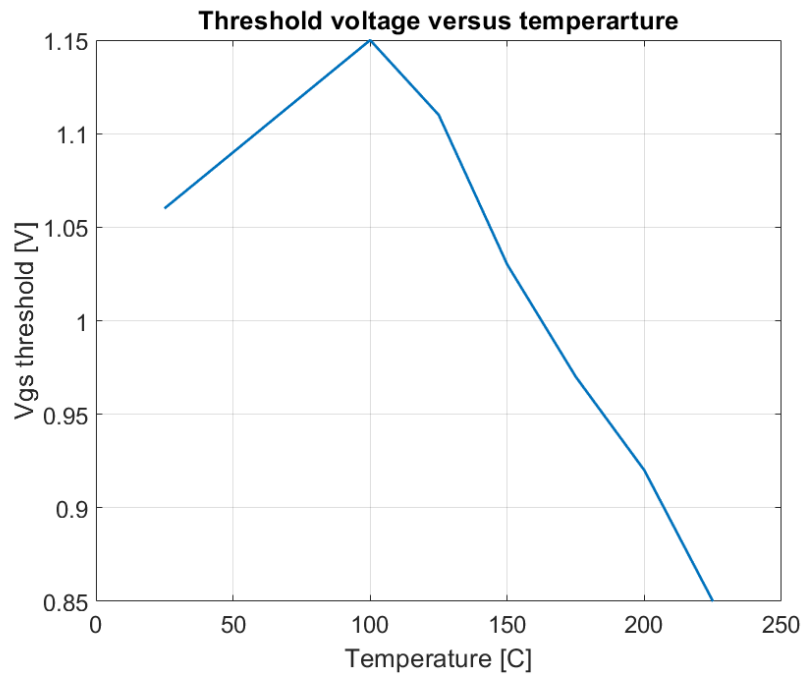

Figure 7: Measured Gate Source Threshold voltage $\left(V_{G S T H}\right)$ in the selected eGaN-FET for various temperatures

\section{Conclusion}

Measurements have shown that the impact of temperature towards parasitic elements in eGaN-FETs is substantial. A large impact can be expected towards dynamic parameters of the device need and therefore need to be taken into account when designing switch-mode power applications. It is noted that the effect of increased output capacitance will impact the switching performance and as a result may effect the lifetime and operation performance of the devices. The output capacitance measurements show an increase of $+18.2 \%$ from $62.4 \mathrm{pF}$ in $100^{\circ} \mathrm{C}$ to $74.1 \mathrm{pF}$ in $225^{\circ} \mathrm{C}$. Comparing this with the datasheet noted values, at a single point of $50 \mathrm{~V}$, the capacitance increase is found to be $+30 \%$ in $225^{\circ} \mathrm{C}$. The reverse transfer capacitance measures a similar response when temperature is increased, but is so small that measurement errors contribute significantly to the total outcome. The impact of temperature is clearly visible which confirms the need for additional work. Threshold voltage was found to be affected by temperature, where up to $100^{\circ} \mathrm{C}$ a rising trend was observed. However, above this temperature the $V_{G S T H}$ showed an declining response with a low of $0.85 \mathrm{~V}$ in $225^{\circ} \mathrm{C}$. A low threshold voltage may lead toward accidental turn-ON events when this device is employed in a switch-mode power converter. 


\section{Acknowledgment}

The authors would like to thank the Danish Innovation Fund for their financial contribution towards this project under reference number 9065- 00005B

\section{References}

[1] J. J. Den Boer, "The use of high temperature electronics in downhole applications," HITEN 1999 - 3rd European Conference on High Temperature Electronics, pp. 149-152, 1999.

[2] A. Szel, Z. Sarkany, M. Bein, R. Bornoff, A. Vass-Varnai, and M. Rencz, "Lifetime estimation of power electronics modules considering the target application," Annual IEEE Semiconductor Thermal Measurement and Management Symposium, vol. 2015-April, pp. 332-335, 2015.

[3] S. Ravyts, W. Van De Sande, M. D. Vecchia, G. Van Den Broeck, M. Duraij, W. Martinez, M. Daenen, and J. Driesen, "Practical considerations for designing reliable DC/DC converters, applied to a BIPV case," Energies, vol. 13, no. 4, pp. 1-21, 2020.

[4] Y. Wang, O. Lucia, Z. Zhang, S. Gao, Y. Guan, and D. Xu, "A Review of High Frequency Power Converters and Related Technologies," IEEE Open Journal of the Industrial Electronics Society, no. September, pp. 1-1, 2020.

[5] P. Tremlett, P. Elliot, and P. Tena, "Origami for Tight Spaces - 3D 250C PCB Assemblies for Control Systems," Ad- ditional Conferences (Device Packaging, HiTEC, HiTEN, and CICMT), vol. 2019, no. HiTen, pp. 000034-000038, 2019.

[6] M. S. Duraij, Y. Xiao, G. Zsurzsan, and Z. Zhang, "Enhancement Mode GaN-FETs in Extreme Temperature Conditions, Part I: Static Parasitic Parameters," HiTEC 2021, Submitted for publication, no. HiTEC, 2021.

[7] EPC, "EPC8010 - Enhancement Mode Power Transistor, Datasheet ," pp. 1-6, 2013.

[8] A. M. Darwish, A. A. Ibrahim, and H. A. Hung, "Temperature dependence of GaN HEMT small signal parameters," International Journal of Microwave Science and Technology, no. December, 2011.

[9] Nexperia, "AN90005 - Understanding Power GaN FET data sheet parameters," vol. Rev. 2.0, pp. 1-21, 2020.

[10] A. Lidow, J. Strydom, M. de Rooij, and D. Reusch, GaN Transistors for Efficient Power Conversion: Second Edition, vol. 9781118844762 . Wiley Blackwell, 2014.

[11] M. J. Tadjer, S. M. Horcajo, T. J. Anderson, R. Cuerdo, K. D. Hobart, and F. Calle, "Temperature and time dependent threshold voltage characterization of $\mathrm{AlGaN} / \mathrm{GaN}$ high electron mobility transistors," Physica Status Solidi (C) Current Topics in Solid State Physics, vol. 8, no. 7-8, pp. 2232-2234, 2011. 